\title{
Prevención y diagnóstico veterinario de la tuberculosis bovina. Una revisión de las tendencias globales
}

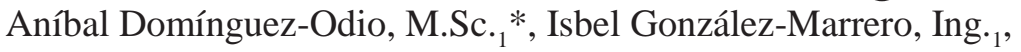
Raiselys Toirac-Proenza, M.Sc. ${ }_{1}$, Yudit Rodríguez-Coipel, M.Sc..$_{1}$

${ }_{1}$ Unidad de Desarrollo e Innovación. Grupo empresarial LABIOFAM, La Habana, Cuba.

Recibido: 10 de septiembre del2016 Aprobado: 4 de octubre del 2018

*Autor de correspondencia:

Cómo citar este artículo: Domínguez-Odio A, González Marrero I, Toirac-Proenza R, Rodríguez-Coipel Y. Prevención y diagnóstico veterinario de la tuberculosis bovina. Una revisión de las tendencias globales. Spei Domus. 2016;12(25):1-16. doi: http://dx.doi.org/10.16925/2382-4247.2016.02.03

Resumen. La tuberculosis bovina (TBb) causada por Mycobacterium bovis es una enfermedad bacteriana grave, crónica, reemergente y zoonótica. Afecta a la producción pecuaria, así como a los animales silvestres y afectivos. En los hatos bovinos provoca sustanciales pérdidas económicas relacionadas con las medidas sanitarias para su control, e importantes restricciones comerciales a los productos derivados. Dado que las estrategias de prevención y control deben actualizarse con relación a los desarrollos científicos, se realizó el estudio para presentar los avances y tendencias científico-tecnológicas alrededor de las estrategias sanitarias para el control de la $\mathrm{TBb}$. Se realizó una amplia consulta de artículos originales y de revisión sobre vacunas y diagnóstico de TBb entre los años 2002 al 2017, en las bases de datos MEDLINE/PubMed,EBSCO y SciELO. Se encontró una activa y consistente acción internacional orientada a explorar formulaciones vacunales y avances en herramientas diagnósticas robustas. No se reportaron avances importantes en el desarrollo de inmunógenos para el ganado bovino. Tampoco se reportó una prueba con altos valores de sensibilidad (Se) y especificidad (Es) para el diagnóstico poblacional de la enfermedad. En consideración a lo anterior, se estima que los adelantos científicos y tecnológicos obtenidos durante los últimos años, no necesariamente van a contribuir por el momento, con el éxito de los programas nacionales de control y erradicación de la TBb. Por lo tanto, en este contexto la priorización de un abordaje integral que considere las realidades de cada región o país, dentro del concepto "Una sola salud", el empleo combinado y estratégico de los sistemas diagnósticos, el firme compromiso político de los gobiernos y la cooperación internacional son indispensables para avanzar en la lucha contra esta enfermedad.

Palabras clave: tuberculosis bovina, Mycobacterium bovis, vacuna, diagnóstico, tendencias. 


\title{
Global trends in the prevention and veterinary diagnosis of tuberculosis bovine
}

\begin{abstract}
Bovine tuberculosis ( $\mathrm{TBb}$ ) caused by Mycobacterium bovis is a serious, chronic, re-emerging and zoonotic bacterial disease. It affects livestock production, as well as wild and affectionate animals. In bovine herds it causes substantial economic losses related to sanitary measures for its control, and important commercial restrictions on derived products. Given that prevention and control strategies should be updated in relation to scientific developments, the study was conducted to present advances and scientific-technological trends around the health strategies for the control of TBb. An extensive consultation of original articles and review of vaccines and TBb diagnosis was carried out between 2002 and 2017, in the MEDLINE / PubMed, EBSCO and SciELO databases. An active and consistent international action was found aimed at exploring vaccine formulations and advances in robust diagnostic tools. No important advances were reported in the development of immunogens for cattle. Also, a test with high sensitivity (Se) and specificity (Es) values for the population diagnosis of the disease was not reported. Considering the above, it is estimated that the scientific and technological advances obtained during the last years, will not necessarily contribute for the moment, with the success of the national $\mathrm{TBb}$ control and eradication programs. Therefore, in this context, the prioritization of an integral approach that considers the realities of each region or country, within the "One health" concept, the combined and strategic use of diagnostic systems, the firm political commitment of governments and international cooperation are indispensable to advance in the fight against this disease.
\end{abstract}

Key words: bovine tuberculosis, Mycobacterium bovis, vaccine, diagnosis, trends.

\section{Prevenção e diagnóstico veterinário da tuberculose bovina. Uma revisão das tendências globais}

Resumo. Introdução: a tuberculose bovina (TBb) causada por Mycobacterium bovis é uma doença bacteriana grave, crônica, reemergente e zoonótica. Afeta a produção pecuária, bem como os animais silvestres e afetivos. Nos rebanhos bovinos, provoca perdas econômicas substanciais relacionadas com as medidas sanitárias para seu controle e importantes restrições comerciais aos produtos derivados. Considerando que as estratégias de prevenção e controle devam ser atualizadas com respeito aos desenvolvimentos científicos, realizou-se este estudo para apresentar os avanços e tendências científico-tecnológicas acerca das estratégias sanitárias para controle da TBb. Metodologia: realizou-se uma ampla consulta a artigos originais e de revisão sobre vacinas e diagnóstico de TBb, entre os anos de 2002 e 2017, encontrados nas bases de dados MEDLINE/PubMed, EBSCO e SciELO. Resultados: encontrou-se uma ação internacional ativa, consciente e orientada a explorar formulações de vacinas e avanços em ferramentas diagnósticas robustas. Não foram reportados avanços importantes no desenvolvimento de imunogênicos para o gado bovino. Tampouco foi reportada uma prova com altos valores de sensibilidade (Se) e especificidade (Es) para o diagnóstico populacional da doença. Conclusões: estima-se que os progressos científicos e tecnológicos obtidos durante os últimos anos não vão, neste momento, contribuir necessariamente com o sucesso dos programas nacionais de controle e erradicação da TBb. Portanto, nesse contexto, a priorização de uma abordagem integral que considere as realidades de cada país ou região (dentro do conceito de "uma única saúde"), o emprego combinado e estratégico dos sistemas diagnósticos, o firme compromisso político dos governos e a cooperação internacional são fatores indispensáveis para avançar na luta contra essa doença.

Palavras-chave: tuberculose bovina, Mycobacterium bovis, vacina, diagnóstico, tendências. 


\section{Introducción}

La tuberculosis bovina ( $\mathrm{TBb}$ ) es una enfermedad crónica, progresiva, debilitante y zoonótica que afecta a los animales domésticos (económicos y afectivos) y silvestres (vida libre y cautiverio). El agente causal es Mycobacterium bovis, una bacteria Gram positiva perteneciente a la familia Mycobacteriaceae y al género Mycobacterium. La enfermedad se caracteriza por lesiones granulomatosas nodulares denominadas tubérculos, que pueden localizarse en cualquier órgano, dado el carácter intracelular obligado del agente causal. La capacidad de $M$. bovis para afectar a diversas especies animales está asociada a la participación combinada de múltiples factores que tienen que ver con las características propias del agente, con el huésped y las complejas variables ambientales [1-4]. La multicausalidad de la enfermedad requiere que se apliquen abordajes integrales como lo propone el concepto de "Una sola salud", formulado por la Organización Mundial de Salud (OMS) y la Organización Mundial de Sanidad Animal (OIE) quienes recomiendan miradas inter y transdisciplinarias, lo mismo que el compromiso de diversos actores en la investigación y en la formulación de políticas públicas sobre sanidad [5].

$\mathrm{El}$ ingreso del agente al individuo susceptible con mayor frecuencia se da por vía oral o respiratoria, con un desarrollo lesional tardío asociado a un periodo de incubación relativamente prolongado [6]. Su confirmación en el rebaño bovino conlleva a graves problemas económicos y sociales, asociados a los gastos por disminución en la producción (carne y leche), al diagnóstico y al sacrificio sanitario. De la misma manera, la pérdida de acuerdos comerciales debido a la imposición de restricciones de movilización, comercialización y exportación del ganado son otros perjuicios relacionados. Por estas razones y su fuerte impacto en la industria ganadera y el comercio internacional, se considera una enfermedad de declaración obligatoria ante la OIE y, por lo tanto, muchos países realizan actividades de vigilancia control y monitoreo, tanto en los hatos bovinos como en animales silvestres [7].

En la actualidad, esta enfermedad zoonótica es un problema sanitario global y re-emerge en un escenario muy complejo $[8,9]$. La deteriorada situación sanitaria está asociada a la acción simultánea de nuevos y múltiples factores, entre los que se destacan la proximidad de poblaciones humanas y animales domésticos con animales silvestres [9]. La dinámica de transmisión intra e interespecies, la estrecha relación genómica con otras micobacterias y la heterogeneidad genética de cepas (epidémicas y endémicas) con diferentes patrones de patogenicidad, virulencia y multiresistencia a fármacos, son otros elementos importantes en este problema. La combinación de estos elementos y otros hacen difícil para muchos países evitar la expansión de la $\mathrm{TBb}$ hacia nuevas zonas o recuperar áreas afectadas [10-15].

Gran parte de las variabilidades genéticas observadas en las cepas circulantes se explica por mecanismos moleculares, principalmente asociadosacambiosgenómicosacumulativos(mutaciones no letales), no siempre dilucidados en profundidad [16]. El aislamiento de cepas letales capaces de generar daño tisular progresivo, acompañada con una disminución de la expresión de interferón gamma (IFN- $\gamma$ ) y radicales libre de nitrógeno (sintetasa de óxido nítrico por macrófagos activados) es un ejemplo de ello [15]. En otros casos, se han detectado mutaciones en los genes $p n c \mathrm{~A}$, inb $\mathrm{R}$, tet $\mathrm{R}$, relacionados con características de mono o multirresistencia a medicamentos antituberculosos como la pirazinamida y niacina empleadas en el control de M. bovis [17].

La situación sanitaria en torno a la TBb puede complejizarse aún más en la medida en que existan brechas en el manejo de fuentes de infección particularmente de los animales silvestres, desconocimientos en algunas regiones y países sobre la prevalencia e incidencia real de la enfermedad y, aún más, por los limitados desempeños de los sistemas de diagnóstico disponibles $[12,18]$.

Las graves implicaciones de la TBb en la salud animal y humana son suficientes motivaciones para buscar con urgencia, soluciones que superen las actuales fallas inmunoprofilácticas, y la ausencia de diagnósticos eficientes. Los resultados obtenidos a nivel internacional, requieren de una constante vigilancia tecnológica por parte de la industria biofarmacéutica veterinaria. Como parte de ese compromiso se realizó la investigación que presenta este artículo, la cual ofrece una visión global y actualizada sobre las tendencias científicas y tecnológicas para la prevención y el diagnóstico de la tuberculosis bovina. 


\section{Desarrollo de vacunas}

En la historia de la tuberculosis y del investigador Robert Koch convergen dos hechos poco comunes en una enfermedad, el descubrimiento del agente causal y el primer fracaso de una vacuna para su control [19]. Han transcurrido muchos años de investigación y aún no se dispone de una formulación inmunoprofiláctica aceptada para bovinos. Sin embargo, el sueño de lograrla no se abandona [20].

En ese sentido, se han realizado grandes avances, entre los cuales, la caracterización genómica completa de $M$. bovis resulta uno de los más relevantes [21]. Se demostró que a pesar de que existe una similitud genómica del $99.95 \%$ de este con M. tuberculosis, subyacen importantes diferencias. La ausencia o modificaciones estructurales de proteínas inducidas por pseudogenes o genes con mutaciones individualizan el comportamiento patogénico, inmunológico y metabólico de $M$. bovis. Sobresalen en tal sentido los genes codificantes de lipoproteínas $(l p p \mathrm{O}, l p q \mathrm{~T}, l p q \mathrm{G}$ y $l p r \mathrm{M})$, proteínas adhesivas e inmunomoduladoras (Rv1759c), proteínas delafamiliaESAT-6(Rv2346c, Rv2347c, Rv3619c, Rv3620c, entre otros), proteínas reparadoras de $\mathrm{ADN}$ ( alkA), enzima glicerol quinasa $(g l p \mathrm{~K})$ entre otras $[21,22]$.

Con relación al hallazgo de inmunógenos potencialmente útiles para vacunas bovinas, se afincan esperanzas en el Bacilo Calmette-Guérin (BCG). Esta opción es favorecida por los bajos costos de producción, amplia experiencia de uso en humanos y genoma conocido. Sin embargo, su aplicación en animales debe superar grandes obstáculos: efectividad variable sin explicación científica en muchos casos, interferencia diagnóstica con la prueba de hipersensibilidad retardada, y la selección de la cepa vacunal adecuada, teniendo en cuenta la variedad existente [7, 23, 24], tal y como se aprecia en la tabla 1.

Una circunstancia que complica su obtención, es la comprobada heterogeneidad de respuestas observadas con las cepas vacunales BCG [24, 25], la variedad de métodos para obtener formulaciones candidatas (vivas e inactivadas, tanto por vía química como por calor) [26] y el enmascaramiento que producen las micobacterias ambientales en la eficacia de BCG [27,28].

Las variaciones de eficacia y seguridad observadas entre cepas BCG a nivel mundial son uno de losaspectosmáspreocupantes, puessonconsecuencias directas de los cambios genéticos ocurridos en su genoma por numerosos pasajes in vitro $[22,25$, 27].Las vacunas BCG Rusia, Japón, Danesa, México, Chicago, Praga, London F10 y Pasteur, difieren entre sí en 28 polimorfismos de un solo nucleótido; más aún la BCG Rusia y México muestran un máximo de 50. Otras importantes variaciones detectadas consisten en el tamaño del genoma y las pérdidas puntuales de nucleótidos. La cepa México 1931 posee un tamaño genómico más pequeño que la Pasteur 1173P2 y Tokio 172, además, tiene dos

Tabla 1. Cepas BCG disponibles a nivel mundial para formulaciones vacunales

\begin{tabular}{cccc}
\hline & \multicolumn{2}{c}{ Cepas vacunales de BCG } & Referencias \\
\hline Denominación & Año de obtención & Vacuna & {$[22,29]$} \\
Brasil & 1924 & BCG-Moreau & {$[22,25,29]$} \\
Rusia & 1924 & BCG-Rusia & {$[22,25,29]$} \\
Tokyo 172 & 1925 & BCG-Japón & {$[29]$} \\
Gothenburg & 1926 & BCG-Suecia & {$[22,25,29]$} \\
Copenhagen 1331 & 1931 & BCG-Danesa & {$[25,29]$} \\
México 1931 & 1931 & BCG-Mexico & {$[25,29]$} \\
Tice & 1934 & BCG-Chicago & {$[25,29]$} \\
Checoslovaquia & 1947 & BCG-Praga & {$[25,29]$} \\
Glaxo 1077 & 1954 & BCG-London F10 & {$[22,25,29]$} \\
Pasteur 1173P2 & 1961 & BCG-Pasteur &
\end{tabular}

Fuente: elaboración propia

Nota: los nombres de las cepas BCG pueden estar conformados por el país, ciudad, laboratorio, año y/o pasajes en la que fueron obtenidas. 
eliminaciones específicas, RDMex02 y RDMex03, que afectan la codificación de proteínas FadD23 y WhiB6. El nivel de virulencia es otro indicador diferenciante entre las cepas BCG. Estudios recientes establecieron un orden de virulencia decreciente entre estas, las cuales fueron clasificadas de la siguiente manera: BCG-Pasteur=BCGChicago $>$ BCG-Rusia $=$ BCG-Moreau $>$ BCG-Danesa $>$ BCG-LondonF10=BCG-Praga=BCG-Japón=BCG-

Suecia $[29,30]$.

La biología molecular proporciona nuevos conocimientos sobre el agente, la relación patógeno-hospedero, y la respuesta inmunológica resultante. Estos conocimientos recientes abren caminos y oportunidades para el desarrollo de nuevas vacunas. En los últimos años se han desarrollado estrategias para lograr una protección efectiva, estable y duradera contra la TBb (figura 1). Se mencionan esfuerzos como el uso de BCG viva e inactivada, con sus variantes de formulación-administración, incluyendo el uso de vacunas vectoriales recombinantes [26, 31, 32], vacunas vivas con atenuación genética [33], y las inductoras de respuesta inmune diferenciante entre animales infectados y vacunados (DIVA) [34].
Los resultados obtenidos con las vacunas anteriores, tanto en modelos experimentales como en animales diana, si bien son prometedores aún tienen un largo camino por recorrer. Todas comparten los mismos retos regulatorios, entre ellos demostrar eficacia, reducción de la transmisibilidad del agente, no interferencia con los medios diagnósticos, estabilidad, duración de la inmunidad, entre otros [31-34].

Ante la ausencia de grandes éxitos en materia de obtención de un buen inmunógeno, se han iniciado nuevos abordajes, entre ellos la proteómica. Identificar y caracterizar nuevas proteínas ha sido de gran ayuda para formular vacunas de subunidad proteica. La intencionalidad en su desarrollo se dirige hacia el logro de una protección mayor y más consistente que la promovida por la BCG. Entre las ventajas de utilizar antígenos independientes en vez de bacterias completas se señalan los mayores niveles de seguridad, eficacia, reproducibilidad y rentabilidad. El punto de partida de estas investigaciones se encuentra en las potencialidades inmunológicas de algunos antígenos proteicos expresados por $M$. bovis, tales como: ESAT-6, CFP10, MPB59, MPB64, MPB70, MPB83, entre otras proteínas [35, 36].

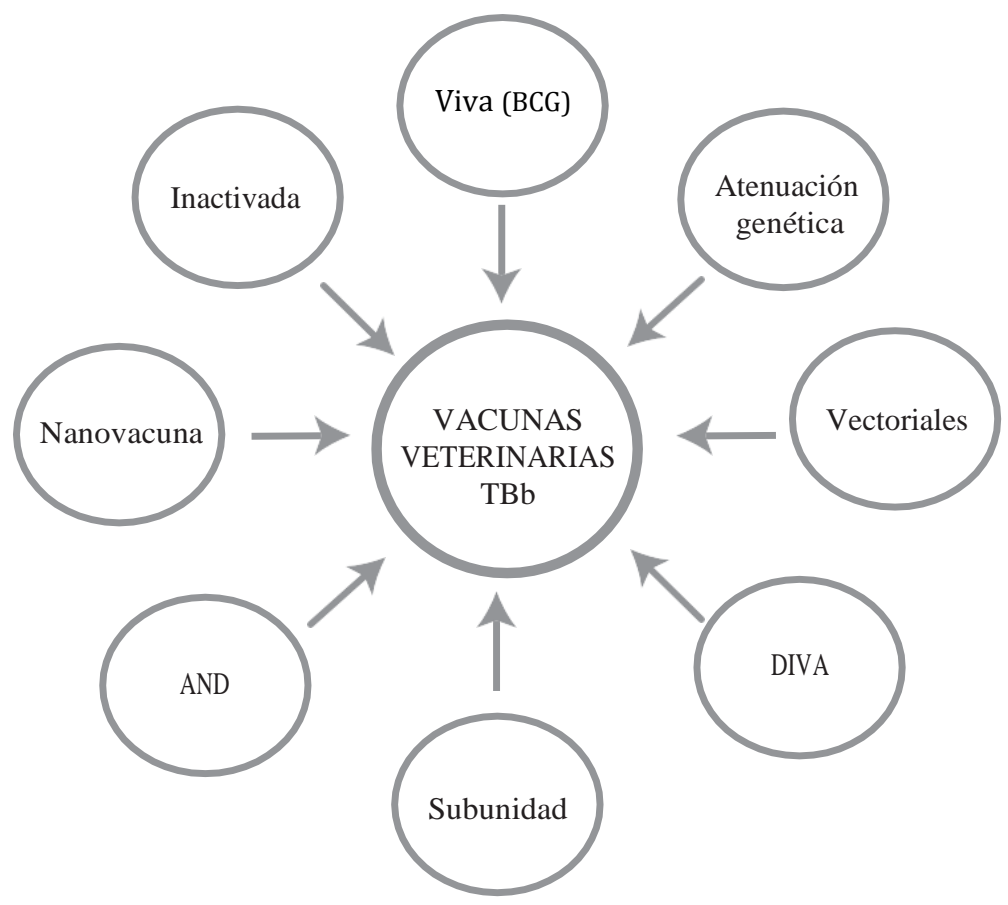

Figura 1. Vacunas veterinarias contra TBb existentes y en desarrollo Fuente: elaboración propia 
Quizás las mayores posibilidades de éxito para este tipo vacunas estén en las proteínas de fusión, obtenidas tras manipular genes específicos. El resultado final es una sola molécula proteica que contenga en su estructura epítopes relevantes de dos o tres subunidades candidatas [35]. Los resultados obtenidos con la proteína de fusión ID83 + adyuvante ISA70 son prometedores. Se demostró que induce una potente e incrementada respuesta humoral $\left(\mathrm{IgG}_{1}\right.$ e $\left.\mathrm{IgG}_{2}\right)$ y celular $(\mathrm{IFN}-\gamma)$ hasta las 8 semanas post-vacunación. Sin embargo, al parecer, no podrá superar la interferencia con la prueba de hipersensibilidad retardada, pues las proteínas utilizadas para la construcción de fusión (Rv1813c, Rv2608 y Rv3620c) son dianas del sistema inmune durante la infección natural con M. bovis [37]. Se añade a esta limitación, las optimizaciones necesarias de las formulaciones (antígeno/adyuvante) y la necesidad de establecer estrategias de vacunación con administración independiente o combinada en animales inmunizados con BCG.

Similar atención científica se le presta al desarrollo de vacunas de subunidad formuladas con filtrado de proteínas y oligonucleótidos específicos, aplicadas solas o combinadas con BCG. Las investigaciones muestran una mayor eficacia y reducción de lesiones tuberculosas en animales inmunizados, cuando se administraron combinadas. En esta línea de pensamiento se ha experimentado además con combinaciones de inmunorreguladores sintéticos, recombinantes y adyuvantes comerciales (Emulsigen凤, Polygen凤). Lo positivo en términos generales de estas experiencias radica en que los valores protectivos medios son significativamente superiores en las combinaciones. Sin embargo, la interferencia con la prueba de hipersensibilidad retardada continúa siendo el obstáculo a vencer, aspecto al parecer resuelto cuando se utilizan Emulsigen凤 (emulsión aceite-agua) y Polygenß (polímero de bajo peso molecular) [38-40].

Una de las estrategias más recientes para el desarrollo de nuevas vacunas es la tecnología de ADN. En teoría, con un número reducido de antígenos se lograría una protección adecuada y segura. Sin embargo, la práctica es mucho más compleja, pues requiere identificar la proteína inmunogénica, aislar su gen, insertarlo en un plásmido de expresión que posea un gen promotor fuerte y garantizar la estabilidad de la transcripción. Desafortunadamente, los candidatos vacunales obtenidos como el plásmido pCMV-83, que contiene el gen mpt83, no compensaron el esfuerzo realizado. Los resultados demostraron que sus niveles protectivos, no exceden a aquella proporcionada por BCG y que requieren administrarse de forma conjunta [41].

Teniendo en cuenta lo anterior, pudieran esperarse resultados prometedores al combinar las vacunas de $\mathrm{ADN}$ con BCG o con péptidos sintéticos, pero la experimentación en la especie dina demuestra que depende de la formulación empleada. Por una parte, se reporta resultados experimentales alentadores con la administración de plásmidos pCMV4.65 y pCMV4.70 codificantes para Hsp 65 y Hsp 70 + BCG Pasteur 1173P2. Esta estrategia de vacunación induce altos niveles de IFN- $\gamma$, reducción de animales portadores de lesiones compatibles con $\mathrm{TBb}$, entre otros parámetros predictivos de inmunidad [40]. Sin embargo, la formulación con péptidos del antígeno Rv3019c + plásmido pCMV301 + adyuvante Emulsigen凤 se comporta de forma contraria [43].

A pesar de estas variabilidades en la respuesta biológica, las vacunas elaboradas a partir de ADN se convierten en candidatas atractivas para la prevención de la tuberculosis bovina, debido a que actúan en presencia de anticuerpos maternos, no exigen cadena de frío y no interfieren con la prueba de tuberculina en los animales. Estas razones también podrían contribuir a una mayor rentabilidad [44]. No obstante, hay que tener en cuenta los inconvenientes prácticos y económicos de múltiples administraciones [40], y los riegos de integración genética siempre presentes al inocular genes de proteínas antigénicas. Se requiere mejorar el diseño de plásmidos más inmunogénicos y que no interfieran con la prueba intradérmica. De la misma manera, es necesario profundizar en el conocimiento de la concentración in vivo en la especie diana de la proteína codificada en el plásmido.

La combinación de la biología molecular con la nanotecnología muestra que, a pesar de ser un camino poco transitado, puede ser una opción factible. Los recientes progresos están relacionados con el desarrollo de nanovacunas con partículas de biopoliester (polihidroxibutirato) combinado con antígenos micobacteriales Ag85A y ESAT-6. Los resultados preliminares en roedores muestran que la respuesta post-vacunal tiene un significativo incremento no solo de IFN- $\gamma$ liberado por CD4 y CD8, sino además de IL-17A, IL-6, TNF- $\alpha$ y IL-2 [45]. Con este enfoque se le presentaría al sistema 
inmune una gran variedad de antígenos, seguramente obtenidos a un costo menor. Estas formulaciones deberán probar, por otra parte, su eficacia en campo, duración de inmunidad y seguridad.

Las tecnologías clásicas de producción de vacunas se mantienen como opción real y práctica, pudiendo utilizarse sus productos en estrategias de vacunación para poblaciones de vida libre [46]. La literatura reporta experiencias con vacunas inactivadas orales y parenterales (adyuvado con ISA $50 \mathrm{~V}$ ) obtenidas a partir de $M$. bovis silvestre. Al parecer su eficacia está mediada por la activación de células $\mathrm{TCD}^{+}$productoras de IFN- $\gamma$, por el complejo mayor de histocompatibilidad clase Iy por células dendríticas [47].

Cuandoestasvacunassonaplicadasaanimales de vida libre, la significativamente baja puntuación de lesiones pulmonares sugiere que podría ser útiles para erradicar la enfermedad del ganado bovino a largo plazo. Las mayores ventajas se encuentran en que su proceso de producción demanda menos costos y se dispone de capacidad industrial instalada para enfrentar su producción segura. Sin embargo, el uso práctico puede verse impedido por la utilización de cepas silvestres circulantes como inóculo primario. Bajo estas condiciones no existen garantías de su estabilidad genética (reversión de la virulencia) y, por tanto, es difícil garantizar la uniformidad y consistencia del producto terminado, así como su efecto biológico (seguridad y eficacia) [48].

\section{Uso de animales de laboratorio}

Un aspecto muy importante a destacar en el estudio de la $\mathrm{TBb}$ es el desarrollo de modelos experimentales. En este ámbito los bovinos como modelo son muy recomendables, aunque su empleo tiene inconvenientes tales como la costosa manutención y la demanda de grandes instalaciones con adecuado nivel de bioseguridad [48].

Contrario a lo que ocurre con la especie bovina, en la cual se tienen experiencias de uso y criterios unificados como modelo experimental, en los animales de vida silvestre la situación es muy compleja. No se cuenta con una especie modelo validada o aceptada de manera unánime. La amplia gama de hospederos posibles demandaría la validación de la prueba para las particularidades de cada caso, pues no es recomendable hacer generalizaciones de los resultados para diferentes especies y situaciones. Lo anterior complica los esfuerzos para estudiar el desempeño de los productos a evaluar y obliga a trabajar caso a caso. No obstante, existen experiencias de trabajo con diversas especies como ratones, cobayos, caprinos y conejos. Ellas cumplen requisitos prácticos y técnicos como su tamaño, fácil manipulación y manutención en condiciones controladas; a la vez que son susceptibles al agente y desarrollan lesiones similares a las observadas en la infección natural. Estos atributos las han convertido en opciones factibles y confiables para futuras investigaciones [15, 41, 49, 50].

\section{Desarrollo de pruebas diagnósticas}

En la práctica clínica veterinaria, el diagnóstico de la $\mathrm{TBb}$ suele ser un proceso largo, costoso y no siempre preciso [51]. Existe en la actualidad una diversidad de técnicas para tipificar [52] y diagnosticar ante o post mortem (clínica, bacteriológica, inmunológica, histopatológica y detección de compuestos orgánicos volátiles), las cuales se resumen en la figura 2 . Se incluye dentro de este arsenal, la inspección macroscópica en faena o frigorífico, como una barrera de protección al consumidor. Sobre la base de las respectivas debilidades y fortalezas reportadas para cada uno de los métodos diagnósticos, algunos autores han sugerido el empleo combinado de algunos de ellos, para cubrir las necesidades diagnósticas que exigen las diversas situaciones epidemiológicas [53-58].

Por su parte la OIE declara como pruebas de referencia el cultivo del microorganismo para confirmar el agente etiológico y la prueba de hipersensibilidad retardada para el diagnóstico en animales vivos (in vivo) y la prescrita para el comercio internacional [59]. Esta técnica desarrollada por Robert Koch [19], mide la reacción local a las 72 h tras la inoculación intradérmica in vivo del derivado proteico purificado (DPP). La tabla 2 presenta algunos laboratorios productores de DPP, junto con las características generales de sus productos, los cuales son comercializados en la región de las américas.

A diferencia de las cepas vacunales, existe un conceso sobre las cepas a utilizar en la obtención del DPP (AN-5/M. bovis y la D4 ER/M. avium) y el método de elaboración. Sin embargo, esta mezcla de proteínas no está plenamente caracterizada y no discrimina si los individuos positivos están sensibilizados por $M$. bovis o por otras micobacterias ambientales. 


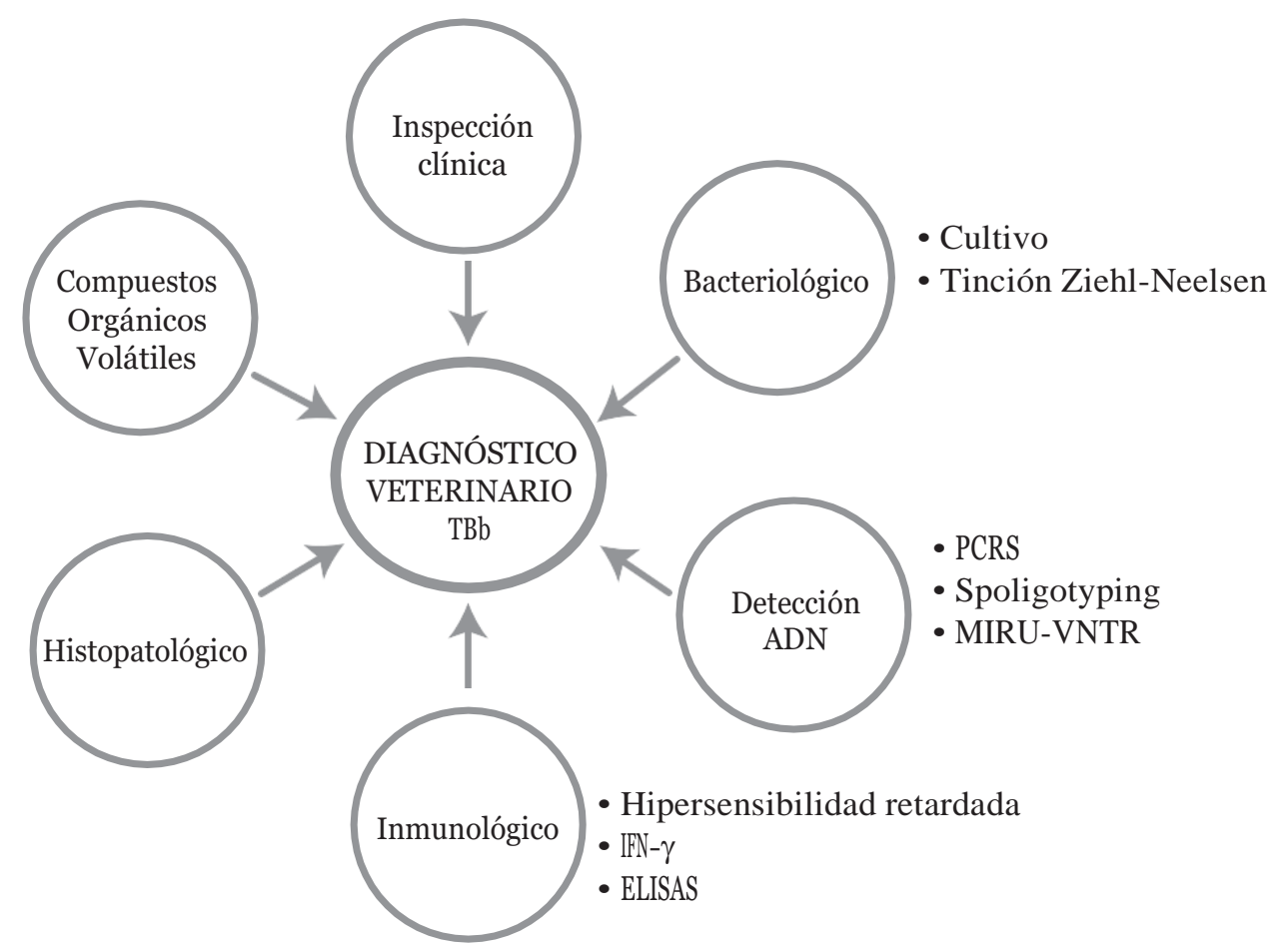

Figura 2. Técnicas diagnósticas y de tipificación, disponibles y en desarrollo para el diagnóstico de TBb Fuente: elaboración propia

Tabla 2. Algunos productores de DPP y características generales del producto comercializado en la región de las Américas

\begin{tabular}{|c|c|c|c|c|}
\hline \multicolumn{5}{|c|}{ Laboratorios productores de DPP } \\
\hline Productor & País & Cepa & $\begin{array}{l}\text { Potencia } \\
\text { (UI/mL) }\end{array}$ & $\begin{array}{l}\text { Presentación } \\
\text { (dosis/bulbo) }\end{array}$ \\
\hline \multirow{2}{*}{ Centro Diagnóstico Veterinario S.A } & \multirow{2}{*}{ Argentina } & AN-5 & 32500 & 100 \\
\hline & & D4 ER & 25000 & 10,20 y 50 \\
\hline MSD Animal Health (InterVet) & Venezuela & AN-5 & 5000 & 20 \\
\hline MEGAVET & Colombia & AN-5 & 32500 & 20 y 50 \\
\hline \multirow{2}{*}{ AsureQuality Limited Upper Hutt } & \multirow{2}{*}{ Nueva Zelandia } & $\mathrm{AN}-5$ & 30000 & 50 \\
\hline & & D4 ER & 25000 & 20 \\
\hline \multirow{2}{*}{ Cooper Zeltia Veterinaria S.A. } & \multirow{2}{*}{ España } & AN-5 & 25000 & 50 \\
\hline & & D4 ER & 25000 & 50 \\
\hline Prionics Lelystad & Holanda & AN-5 & $2000-4875$ & 50 \\
\hline \multirow{2}{*}{ LABIOFAM } & \multirow{2}{*}{ Cuba } & $\mathrm{AN}-5$ & 32500 & 100 \\
\hline & & D4 ER & 7500 & 50 \\
\hline
\end{tabular}

Fuente: elaboración propia 
La intradermorreacción está mediada por la respuesta inmune celular del huésped. Algunos trabajos reportan las debilidades de esta técnica en términos de su baja sensibilidad (72\% -91\%), y especificidad (78\% - 96\%) la doble manipulación de los animales, y la incapacidad para detectar animales con respuesta inmune disminuida [53, 56, 57, 60]. No obstante, hasta que se llegue a un consenso mundial y oficial sobre su sustitución, continuará como el método estándar y de referencia, contra el cual se comparan los métodos de diagnóstico en desarrollo [54, 55].

Los estudios proteómicos de $M$. bovis realizados en años recientes, han incrementado el número de proteínas identificadas, purificadas y caracterizadas por sus potencialidades diagnósticas [21-22]. Algunas de ellas han sido evaluadas, como es el caso de ESAT-6, CFP-10 Rv-3615c, EsxI, Mb0143, PE5, PE13, MPB70, MPB83. El uso propuesto para estas moléculas recombinantes tanto de manera individual como combinada, se orientan en muchos casos hacia el diagnóstico DIVA e intradérmico. Entre las razones para su elección se menciona la existencia de caracterización y estandarización bien establecida para cada una de ellas. Se aspira que puedan aplicarse en diferentes áreas (endémicas, así como en baja y alta prevalencia) y variedad de animales, para diferenciar animales sensibilizados naturalmente por $M$. bovis; de aquellos sensibilizados por otras micobacterias algunas de ellas ambientales, y no asociadas con la enfermedad en bovinos. Los resultados no parecen estar muy cercanos, las mayores posibilidades están ubicadas en los antígenos ESAT-6, CFP-10, EsxI y PE5, aunque todavía no existen suficientes evidencias para considerar el remplazo de la DPP [61-63].

Otros esfuerzos están dirigidos a refinar, automatizar y agilizar el diagnóstico, mediante la detección de anticuerpos circulantes específicos por ELISA. Esta técnica puede encontrar animales afectados aún en la fase subclínica, así como la confirmación ante mortem de la enfermedad. Las proteínas MB2515c, MB1895c, y MB1554c (Pks5), MPB70 y MPB83 son algunas de las candidatas para su uso diagnóstico, siendo las dos últimas las de experiencia de uso [64-67]. Las ventajas de estos sistemas se encuentran en la posibilidad de resultados muy rápidos en pocas horas, evalúan respuesta inmune, son más fáciles de estandarizar, y demandan menos reactivos y equipamientos que el sistema de amplificación de ácidos nucleicos, mediante la reacción en cadena de la polimerasa (PCR).

Sin embargo, se requieren estudios adicionales más profundos para conocer cuándo es mejor usarlos, debido a variabilidades diagnósticas asociadas a tiempo de exposición a $M$. bovis/PPD, infecciones con micobacterias y tipo de formulación. Los sistemas que emplean ambas moléculas por separado reportan en las 3 primeras semanas postsensibilización con DPP, bajos valores de sensibilidad (21,8\%) y especificidad (77,7\%) [64].El empleo combinado mejora estos indicadores hasta un 63 y $98 \%$ a las 14 semanas posexposición experimental a $M$. bovis. La limitación mayor radica en la reactividad variable frente a $M$. kansasii, asociado a la elevada similitud existente (81 y 77\%)con laMPB70y MPB83 de M. bovis [67]. Sin embargo, esta reactividad cruzada es minimizada cuando se emplea MB1554c, no solo para esta micobacteria sino además para $M$. avium subespecie sp. paratuberculosis [64].

La inclusión de más antígenos o sus fragmentos (ESAT6, CFP-10, PPDb y péptidos de MPB70), conjuntamente con MPB70 y MPB83, no supera las expectativas en cuanto a incrementos significativos del desempeño diagnóstico. Estudios recientes demostraron que este tipo de ELISA posee baja sensibilidad $(32,6 \%)$ y alta especificidad $(98,3 \%)$ en rebaños libres e infectados naturalmente. En rebaños con diferentes estadios de la infección (inicial y crónica) y sensibilizados con DPP, se obtuvieron pobres resultados pudiendo detectar como positivo solo el 85,2 y $83,3 \%$ de los animales investigados [58]

Mención independiente merecen los ELISAs DIVA, formulados con interleuquinas (2, y 17A) procedentes de células $\mathrm{T}$ multifuncionales [68, 69] y combinaciones de MPB70-MPB83 con ESAT6-CFP-10, estas últimas ausentes en micobacterias ambientales y cepas de BCG. No obstante, a la enorme utilidad en la práctica clínica su uso está limitado en un inicio por la no elección de una cepa vacunal definitiva [61].

De manera paralela se realizan esfuerzos para medir la inmunidad humoral en los animales enfermos. Uno de ellos consiste en inducir las inmunoglobulinas A y $\mathrm{G}(\operatorname{IgA}$ y $\mathrm{IgG})$ con los antígenos MPB70 y MPB83 procedentes de la cepa BCGJapón. Los resultados evidencian que la IgA posee mejor desempeño para ambos antígenos en cuanto a sensibilidad (MPB70: 100\%, MPB83: 96,7\%) y especificidad(MPB70:93,3\%, MPB83: 100\%) que la IgG 
(sensibilidad MPB70: 90\%, MPB83: 93,3\% y especificidad MPB70: 93,3\%, MPB83: 100\%). Este hecho supone una nueva y atractiva herramienta para el diagnóstico, la cual, por su simplicidad, flexibilidad y beneficios económicos, tiene posibilidad de ser usado como un diagnóstico complementario [70]. Sin embargo, la baja utilidad para la detección de otros antígenos como Rv1483c y PstS1 es una preocupación importante.

En materia de identificación de $\mathrm{TBb}$, también se incursiona en el empleo de marcadores genómicos, los cuales después de una secuenciación y comparación en distintas bases de datos, permiten refinar el diagnóstico [71]. La discriminación entre las micobacterias (complejo tuberculoso y ambientales) mediante marcadores moleculares es relevante desde el punto de vista clínico, si se tiene en cuenta la elevada similitud genética existente. Sobresalen el uso combinado de la región $16 \mathrm{~S}$ ARNr, la secuencia de inserción IS6110 y las regiones genómicas RD1 (Rv3875-esat6), RD4 (Rv1510) y RD9 (Rv2073c), para detectar micobacterias del complejo tuberculoso y no tuberculoso [72]. Otras combinaciones de marcadores son reportadas por la literatura, entre ellas 16S ARNr, rpoB y hsp64 para detectar micobacterias no tuberculosas, con resultados superiores a Speed-Oligol Mycobacteria (16S ARNr) y GenoTypel Mycobacterium (23S ARNr) presentes en el mercado [73].

Estos marcadores pueden usarse en una amplia variedad de especímenes (aislados clínicos, tejidos frescos o conservados) [71-73]. No obstante, estas técnicas moleculares son poco adecuadas para diagnosticar M. bovis de forma rutinaria y con grandes cantidades de muestras. En el caso particular del marcador IS6110, la gran cantidad de ADN purificado en condiciones de alta calidad exige disponer de cepas viables y obliga a que el tiempo de obtención del genotipo se prolongue considerablemente. Influyen en este hecho, los costos por muestras trabajadas, resultados variables asociados al tipo de muestras, ciclos de amplificación ineficientes, entre otros aspectos.

Uno de los métodos moleculares más usados para la tipificación de cepas de M. bovis es la espoligotipificación (Spoligotyping), que es muy útil en estudios de epidemiología molecular y diferenciación de cepas pertenecientes al complejo $M$. tuberculosis. Tienen como ventajas, la fácil elaboración, poca cantidad de ADN requerido, robustos y altamente reproducibles. Más que eso, la espoligotipificación dispone de una base de datos internacional (www.mbovis.org) con más de 11.000 patrones de aislamientos, obtenidos en más de 90 países. Su acceso on line permite de forma global reportar nuevos aislamientos, utilizar nomenclatura estándar y conocer adicionalmente regiones afectadas, especie, fin zootécnico, cepas circulantes con patrones genéticos comunes y asociaciones de aislamientos entre animales y humanos [74-77].

La disponibilidad de estos datos demostró la variedad genotípica en las fincas ganaderas de América y sus relaciones epidemiológicas con sus similares de Europa. En Brasil, por ejemplo, los espoligotipos SB0295, SB121,SB1055,SB1145 y SB140 reportados en los estados de Bahía, São Paulo y Minas Gerais, están presentes también en países como Argentina, México, Inglaterra, España, Portugal y Francia [76]. Estudios similares realizados en Colombia evidenciaron, por su parte, la circulación de los genotipos, SB1075, SB0822, SB0121, SB0961, SB0295, SB1190 y SB0120 en bovinos y búfalos de los departamentos de Antioquia, Boyacá, Cundinamarca y Magdalena, que había sido identificado previamente en Reino Unido, mientras que los SB0822 y SB0121 lo fueron en Holanda, España, Brasil y México [77] y el SB0120 en Francia, desde 1978 [13], y Argentina [74]. Estos resultados son de enorme utilidad epidemiológica, pues demuestran las consecuencias sanitarias de la movilización de animales falsos negativos, además de la amplia y activa distribución de la TBb, tanto en países desarrollados, como en vías de desarrollo.

Otra estrategia utilizada para establecer vínculos entre variabilidad genética de $M$. bovis y su dispersión en la población de hospedadores (epidemiología molecular) es la identificación del número variable de repeticiones en tándem [13, 74]. Los beneficios de su uso están asociados con la posibilidad de distinguir cepas relacionadas epidemiológicamente, comparar los resultados con otros laboratorios de países con escenarios epidemiológicos diferentes y no requerir grandes cantidades de ADN. Su empleo en ciervos permitió demostrar que los loci MIRU4, ETRA, QUB11b y Mtub4 muestran elevada capacidad discriminatoria para diferenciar las distintas micobacterias que afectan a esta especie silvestre [78]. Esta técnica ha sido de mucha utilidad para las zonas ganaderas que comparten fronteras comunes. Al conocer el perfil genético y el grado de relación genética de las cepas circulantes en la región por medio de los loci ETR-A, QUB11a, QUB-11b, QUB-3232 y QUB-3336, México estáen 
condiciones de detectar, trazar e identificar el lugar de origen de un brote [79].

Los métodos de amplificación de ácidos nucleicos mediante PCR detectan de forma rápida M. bovis en muestras clínicas primarias (exudados nasales, entre otros) y tejidos (ganglios linfáticos de cabeza y tórax, entre otros) sin necesidad de cultivo previo [80, 81]. La principal desventaja de estos sistemas radica en que diagnostican falsos positivos con mucha facilidad debido principalmente a la contaminación de los reactivos en el proceso del laboratorio. Aun así, el diagnóstico de $\mathrm{TBb}$ mediante las variantes de PCR se ha convertido en una de las técnicas más rápidas, seguras y confiables [82-84].

Las técnicas moleculares, si bien permiten la diferenciación de cepas de $M$. bovis, y proporcionan información sobre la fuente de infección, rutas de transmisión y reservorios de forma rápida y certera, tienen como limitante su alto costo económico. La logística y sustentabilidad en el tiempo son problemas asociados a estas técnicas, que limitan su uso a laboratorios especializados, validados y centralizados. Por estas razones, el uso del cultivo micobacteriano no parece que pueda serfácilmente sustituido por el momento, en los países con economías en vías de desarrollo.

Por último, es necesario indicar que los importantes avances en el conocimiento de la respuesta del huésped y sus diversos componentes han permitido el surgimiento del diagnóstico por compuestos orgánicos volátiles. Este tipo de diagnóstico identifica animales afectados y vacunados, a través de los perfiles de compuestos específicos (aldehídos, alcanos, cetonas, alcoholes, y derivados del benceno). Lo no invasivo de su práctica, fácil muestreo (heces y aliento exhalado), potencialidad DIVA y posibilidad de uso en animales silvestres son las principales fortalezas que lo caracterizan [85]. Sin embargo, los equipos costosos (cromatografía de gases/espectrometría de masas y nanomateriales), la alta experticia para manipularlos, la necesidad de patrones y la obligatoriedad de realizar técnicas para pre-concentrar y deshumificar las muestras son las principales debilidades prácticas, que muy seguramente impedirán su uso generalizado. Por lo pronto, se requieren nuevos estudios en poblaciones experimentales y naturalmente infectadas en zonas con diferentes comportamientos epizootiológicos, para verificar o refinar los patrones de biomarcadores [86].

\section{Conclusiones}

1. La creciente información especializada en los últimos años sobre $\mathrm{TBb}$ revela que existe una acción consolidada a nivel mundial para buscar de forma prioritaria formulaciones vacunales y diagnósticas robustas.

2. Las vacunas candidatas ensayadas carecen de antígenos apropiados, así como de formulaciones y esquemas de administración adecuados para inmunizar bovinos de forma eficaz, duradera y diferenciante. Teniendo en cuenta que el paso de los estudios experimentales a los ensayos clínicos de campo requiere definir la cepa vacunal, y disponer de evidencias sobre seguridad, potencia, estabilidad, duración de inmunidad e inmunidad esterilizante, es muy probable que en los próximos 5 años no se den avances relevantes sobre este tema.

3. No se ha desarrollado una formulación que permita diagnosticar el verdadero estado de infección o enfermedad en los animales. Por esta razón se prevé que el diagnóstico rutinario de la $\mathrm{TBb}$ continúe empleando combinaciones de ensayos.

4. El éxito de los actuales programas nacionales de control y erradicación no depende de los adelantos científicos logrados, o por alcanzar. La pieza clave está en combinar los sistemas diagnósticos disponibles según las realidades de cada región o país, pero sobre todo en el enfoque sanitario integral (una sola salud) de la enfermedad, en el firme compromiso político de los gobiernos, y en la cooperación internacional para erradicarla.

\section{Bibliografía}

[1] Ramdas KE, Lyashchenko KP, Greenwald R, Austerman S, McManis C, Waters WR. Mycobacterium bovis infection in humans and cats in same household, Texas, USA, 2012. Emerg. Infect. Dis. 2015; 21(3): 480-3. Disponible en: https://dx.doi.org/10.3201/ eid2103.140715

[2] MusokeJ,Hlokwe T,MarcottyT,duPlessis BJ,Michel AL. Spillover of Mycobacterium bovis from wildlife to livestock, South Africa. Emerg. Infect. Dis. 2015; 21(3): 448-51. doi:10.3201/eid2103.131690.

[3] Müller B, Dürr S, Alonso S, Hattendorf J, Laisse C. Zoonotic Mycobacterium bovis induced tuberculosis in humans. Emerg. Infect. Diseas. 2013; 19(6): 899-908. doi:10.3201/eid1906.120543. 
[4] Estrada CG, Valencia AS, Octavio VW. Prevalencia de tuberculosis en primates en cautiverio en el municipio de Florencia, Caquetá. Rev. Med. Vet. Zootec. 2011. 6(2): 45-52.

[5] Travis DA, Sriramarao P, Cardona C, Steer CJ, Kennedy S, Sreevatsan S. One Medicine One Science: a framework for exploring challenges at the intersection of animals, humans, and the environment. Ann. N. Y.Acad. Sci. 2014; 1334(1):26-44. Disponible en: http://dx.doi.org/10.1111/nyas.12601

[6] Pesciaroli M, Álvarez J, Boniotti MB, Cagiola M, Di Marco V, Marianelli C. Tuberculosis in domestic animal species. Res. Vet. Sci. 2014; 97 Suppl: S78-85. doi: https://doi.org/10.1016/J.RVSC.2014.05.015

[7] Buddle BM, de Lisle GW, Griffin J, Hutchings SA. Epidemiology, diagnostics, and management of tuberculosis in domestic cattle and deer in New Zealand in the face of a wildlife reservoir. N. Z. Vet. J. 2015: 1-9. Disponible en: http://doi/full/10.1080/ 00480169.2014.929518

[8] El-Sayed A, El-Shannat S, Kamel M, Castañeda MA, Castañeda H. Molecular Epidemiology of Mycobacterium bovis in Humans and Cattle. Zoonoses Public Health. 2016; 63(4):251-64. Disponibleen: http://dx.doi.org/10.1111/zph.12242

[9] Rabozzi G, Bonizzi L, Crespi E, Somaruga C, Sokooti M, Tabibi R, Vellere F, et al. Emerging zoonoses: the "one health approach". Saf Heal Work. 2012; 3(1): 77-83. Disponible en: https://doi.org/10.5491/ SHAW.2012.3.1.77

[10] Hlokwe TM, van Helden P, Michel AL. Evidence of increasing intra and inter-species transmission of Mycobacterium bovis in South Africa: are we losing the battle? Prev. Vet. Med. 2014; 115(12):10-7. Disponible en: https://doi.org/10.1016/j. prevetmed.2014.03.011

[11] Sanou A, Tarnagda Z, Kanyala E, Zingué D, Nouctara M, Ganamé Z. Mycobacterium bovis in Burkina Faso: epidemiologic and genetic links between human and cattle isolates. PloS One. Negl. Trop. Dis. 2014; 8(10): e3142. Disponible en: https://doi. org/10.1371/journal.pntd.0003142

[12] Ortiz ME. La Tuberculosis Bovina: un problema aún sin resolver. Rev Iber Cien Biol Agrop. 2015; 4(8).

[13] Hauer A, De Cruz K, Cochard T, Godreuil S, Karoui C, Henault S, et al. Genetic evolution of Mycobacterium bovis causing tuberculosis in livestock and wildlife in France since 1978.PLoS One. 2015;10(2): e0117103. Disponible en: https://doi.org/10.1371/ journal.pone.0117103

[14] de Souza FA, Luiza AR, Gonçalves J, Ribeiro A, Eugênio S, Pires A, Alves G, et al. Genetic profiles of Mycobacterium bovis from a cattle herd in southernmost Brazil. Sem Ciên Agrár. 2016; 37 (5): 3719-3726. Disponible en: http://dx.doi.org/10.5433/1679-0359.2016v37n5Supl2p3719

[15] Aguilar LD, Zumárraga M J, Jiménez OR, Gioffré AK, Bernardelli A, Orozco EH, et al. Mycobacterium bovis with different genotypes and from different hosts induce dissimilar immunopathological lesions in a mouse model of tuberculosis. Clin. Exp. Immunol. 2009. 157: 139-147. Disponible en: https://doi:10.1111/j.1365-2249.2009.03923.x

[16] Millán LM, Jiménez M, Martín C, Samper S. In-depth analysis of the genome sequence of a clinical, extensively drug-resistant Mycobacterium bovis strain. Tuberculosis. 2016; 100: 46-52. Disponible en: https://doi.org/10.1016/j.tube.2016.06.005.

[17] Min Y, Chun HG, Jialing H, Lei Z, Qiaoyun H, Zheng GH. InbR, a TetR family regulator, binds with isoniazid and influences multidrug resistance in Mycobacteriumbovis BCG. Sci.Rep. 2015;5:1-14. Disponible en: https://doi.org/10.1038/srep13969.

[18] Ramos DF. Silva PE. Dellagostin OA. Diagnosis of bovine tuberculosis: review of main techniques. Braz. J. Biol. 2015; 75(4): 830-37. Disponible en: http://dx.doi.org/10.1590/1519-6984.23613.

[19] Blevins SM, Bronze MS. Robert Koch and the golden age of bacteriology. Int. J. Infect. Dis. 2010; 14(9): e744-e751. Disponible en: https://doi.org/10.1016/j. ijid.2009.12.003.

[20] Conlan AJ, Pollock EB, McKinley TJ, Mitchell AP, Jones GJ. Potential benefits of cattle vaccination as a supplementary control for bovine tuberculosis. PLoS. Comput. Biol. 2015; 11(2): e1004038. Disponible en: https://doi.org/10.1371/journal. pcbi. 1004038 .

[21] Garnier T, Eiglmeier K, Camus J, Medina N, Mansoor H, Pryor, et al. The complete genome sequence of Mycobacterium bovis. PNAS. 2003; 100(13): 7877 7882.

[22] Ritz N, Curtis N. Mapping the global use of different BCG vaccine strains. Tuberculosis. 2009; 89(4):24851. Disponible en: http://doi.org/10.1016/j. tube.2009.03.002.

[23] Pym AS, Brodin PB, Roland H, Stewart T. Loss of RD1 contributed to the attenuation of the live tuberculosis vaccines Mycobacterium bovis BCGand Mycobacterium microti. Molecular Microbiology. 2002; (46)3: 709-717. Disponible en: https://doi. org/10.1046/j.1365-2958.2002.03237.x.

[24] Villena M.R. BCG 1948-2014: ¿La misma cepa? Neumol. Pediatr. 2015; 10 (4): 189 - 193.

[25] Copin R, Coscollá M, Efstathiadis E, Gagneux $\mathrm{S}$, Ernst JD. Impact of in vitro evolution on antigenic diversity of Mycobacterium bovis bacillus 
Calmette-Guerin (BCG). Vaccine. 2014; 32(45): 5998-6004. Disponible en: http://doi.org/10.1016/j. vaccine.2014.07.113

[26] Van der Heijden, Chileshe J, Vernooij C, Gortazar C, Juste A, Sevilla I, Crafford E, et al. Immune response profiles of calves following vaccination with live BCG and inactivated Mycobacterium bovis vaccine candidates. PLoS One. 2017; 12(11): e0188448. Disponible en: https://doi.org/10.1371/journal. pone. 0188448

[27] Fine PE. Variation in protection by BCG: implications of and for heterologous immunity. Lancet. 1995;346(8986):1339-45. Disponible en: https:// doi.org/10.1016/S0140-6736(95)92348-9

[28] Stanford JL, Shield MJ, Rook GA. How environmental mycobacteria may predetermine the protective efficacy of BCG. Tubercle 1981;62(1):55-62.

[29] Orduña P, Cevallos MA, de León SP, Arvizu A, Hernández GL, Mendoza HG, López VY. Genomic and proteomic analyses of Mycobacterium bovis BCG Mexico 1931 reveal a diverse immunogenic repertoire against tuberculosis infection. BMC Genomics. 2011; 12:493. Disponible en: http://doi. org/10.1186/1471-2164-12-493

[30] Zhang L, Ru H, Chen F, Jin C, Sun R, Fan, X, Liu J, et al. Variable virulence and efficacy of BCG vaccine strains in mice and correlation with genome polymorphisms. Mol Ther. 2016; 24(2): 398-405. Disponible en: http://doi.org/10.1038/mt.2015.216.

[31] Dean G, Whelan A, Clifford D, Salguero FJ, Xing $\mathrm{Z}$, Gilbert S, et al. Comparison of the immunogenicity and protection against bovine tuberculosis following immunization by BCG-priming and boosting with adenovirus or protein based vaccines. Vaccine. 2014; 32(11):1304-10.

[32] Dean G, Clifford D, Gilbert S, McShane H, Hewinson RG, Vordermeier HM, et al. Effect of dose and route of immunisation on the immune response induced in cattle by heterologous Bacille Calmette-Guerin priming and recombinant adenoviral vector boosting. Vet Immunol Immunopathol. 2014; 158(3-4):208-13.

[33] Blanco FC, Bianco MV, Garbaccio S, Meikle V, Gravisaco MJ, Montenegro V, et al. Mycobacterium bovis $\Delta$ mce2 double deletion mutant protects cattle against challenge with virulent $M$. bovis. Tuberculosis. 2013; 93(3):363-72. Disponible en: https://doi. org/10.1016/j.tube.2013.02.004

[34] Vordermeier M, Jones GJ, Whelan AO. DIVA reagents for bovine tuberculosis vaccines in cattle. Expert. Rev. Vaccines. 2011;10(7):1083-91. Disponible en: https://doi.org/ 10.1586/erv.11.22
[35] Lin JM, Wang CF, Guan JN, Ma HX, Hou S, Liu X, Jiang XY. Fusion expression on the esat- 6 and cfp10 genes of Mycobacterium bovis in Escherichia coli. Appl. Mech. Mat. 2013; 421: 354-358.

[36] Hope JC, Villarreal RB. Aspects of vaccine development. Bovine TB and the development of new vaccines. Comp. Immunol. Microbiol. Infect. Dis. 2008; 31(2-3): 77-100. Disponible en: https://doi. org/10.1016/j.cimid.2007.07.003

[37] Jones GJ, SteinbachaS, CliffordbD,BaldwincSL, Iretonc GC, Colerc RN. Reedc SG, Vordermeiera HM. Immunisation with ID83 fusion protein induces antigen-specific cell mediated and humoral immune responses in cattle. Vaccine. 2013; 31(45): 5250 5255. https://doi.org/10.1016/j.vaccine.2013.08.051.

[38] Wedlock DN, Skinner MA, de Lisle GW, Vordermeier HM, Hewinson RG, Hecker R, S. Littel-van DH, Babiuk LA, Buddle BM. Vaccination of cattle with Mycobacterium bovis culture filtrate proteins and $\mathrm{CpG}$ oligodeoxynucleotides induces protection against bovine tuberculosis. Vet. Immun. Immunopath. 2005; 106 (1-2): 53-63. Disponible en: https:// doi.org/10.1016/j.vetimm.2005.01.002

[39] Wedlock DN, Denis M, Skinner MA, Koach J, de Lisle GW, Vordermeier HM, Hewinson RG, et al. Vaccination of cattle with a $\mathrm{CpG}$ oligodeoxynucleotide-formulated mycobacterial protein vaccine and Mycobacterium bovis BCG induces levels of protection against bovine tuberculosis superior to those induced by vaccination with BCG alone. Infect. Immun. 2005; 73 (6): 3540-46. Disponible en: https:// doi.org/10.1128/IAI.73.6.3540-3546.2005

[40] Skinner MA, Buddle BM, Wedlock N, Keen D, de Lisle GW, Tas RE, et al. A DNA prime: BCG boost vaccination strategy in cattle induces protection against bovine tuberculosis. Infect Immun 2003;71 (9):4901-7. Disponible en: https://doi.org/10.1128/ IAI.71.9.4901-4907.2003.

[41] Chambers MA, Williams A, Hatch G, Gavier WD, Hall G, Huygen K, Lowrie D. et al. Vaccination of guinea pigs with DNA encoding the mycobacterial antigen MPB83 influences pulmonary pathology but not hematogenous spread following aerogenic infection with Mycobacterium bovis. Infect. Immun. 2002, 70 (4): 2159-2165. Disponible en: https://doi. org/10.1128/iai.70.4.2159.2165.2002.26

[42] Cai H., Yu D.H., Hu X.D., Li S.X., Zhu Y.X. A Combined DNA vaccine-prime, BCG-boost strategy results in better protection against Mycobacterium bovis challenge. DNA Cell Biol. 2006, 25(8): 438-447. Disponible en: https://doi.org/10.1089/ dna.2006.25.438 
[43] Vordermeier HM, Pontarollob R, Karvonenb B, Cocklea P, Heckerc R, Singhd M, Babiukb LA, Hewinsona RG, Littel-van den Hurk SD. Synthetic peptide vaccination in cattle: induction of strong cellular immune responses against peptides derived from the Mycobacterium bovis antigen Rv3019c. Vaccine. 2005; 23(35): Disponible en: 4375-4384. https://doi.org/10.1016/j.vaccine.2005.04.022

[44] Buddle BM. Tuberculosis vaccines for cattle: the way forward. Expert Rev. Vaccine. 2010; 9 (10):11211124. Disponible en: http://dx.doi.org/10.1586/ erv.10.112

[45] Parlane NA, Rehm BH, Wedlock DN, Buddle BM. Novel particulate vaccines utilizing polyester nanoparticles (bio-beads) for protection against $M y$ cobacterium bovis infection-a review. Vet. Immun. Immunopath. 2014; 158(1-2):8-13. Disponible en: https://doi.org/10.1016/j.vetimm.2013.04.002

[46] Garrido JM, Sevilla IA, Beltra 'n-Beck B, Minguijo'n E, Ballesteros C. Protection against tuberculosis in eurasian wild boar vaccinated with heat inactivated Mycobacterium bovis. PLoS One. 2011; 6: e24905. Disponible en: https://doi.org/ 10.1371/ journal.pone.0024905

[47] Beltrán BB, de la Fuente J, Garrido JM, Aranaz A, Sevilla I, Villar M, Boadella M. Oral vaccination with heat inactivated Mycobacterium bovis activates the complement system to protect against tuberculosis. PLoS One. 2014; (9): 5, e98048. Disponible en: https://doi.org/10.1371/journal.pone.0098048

[48] Villarreal RB, Berg S, Chamberlain L, McShane $\mathrm{H}$, Hewinson R, Clifford D. Development of a BCG challenge model for the testing of vaccine candidates against tuberculosis in cattle. Vac. 2014; 32(43):5645-9. Disponible en: https://doi. org/10.1016/j.vaccine.2014.08.009.

[49] Ramires IC, Santillán FM, Arellano RB, Morales AF, Tenorio GV. Detección de secuencia nucleotídica de Mycobacterium bovis a partir de ADN de moco nasal de caprinos inoculados experimentalmente. Vet. Mex. 2006; 37(2): 191-196.

[50] Nedeltchev GG, Raghunand TR, Jassal MS, Lun S, Cheng QJ, Bishai1 WR. Extrapulmonary dissemination of Mycobacterium bovis but not Mycobacterium tuberculosis in a bronchoscopic rabbit model of cavitary tuberculosis. Infect. Immun. 2009; 77(2): 598-603. Disponible en: https://doi.org/10.1128/ IAI.01132-08.

[51] Garbaccio S, Barandiaran S, Fernandez A, Macias A, Magnano G, Martínez V, Peyrú M, et al. Ensayo interlaboratorio: aislamiento de Mycobacterium bovis a partir de lesiones granulomatosas en bovinos. Rev. Arg. Microb. 2016; 48(2):161-165. Disponible en: http://dx.doi.org/10.1016/j.ram.2016.03.004.
[52] Acosta RS, Estrada CC, Milián FS. Tipificación de cepas de Mycobacterium bovis. Revisión. Téc. Pecu. Méx. 2009; 47(4): 389-412.

[53] Díaz OF, Banda RV, Jaramillo LM, González SD, Estrada CC. Identificación de bovinos portadores de Mycobacterium bovis aplicando técnicas inmunológicas y moleculares. Vet. Mex. 2003; 34 (1): 13-26.

[54] Furlanetto LV,Figueiredo ES, Conte CA Ricardo CT, Silva FG, Silva JT, Lilenbaum W, et al. Uso de métodos complementares na inspeção post mortem de carcaças com suspeita de tuberculose bovina. Pesq. Vet. Bras. 2012.32(11): 45-52. Disponible en: http:// dx.doi.org/10.1590/S0100-736X2012001100011.

[55] Seva J, Sanes JM, Ramis G, Mas A, Quereda JJ, Villarreal RB. Evaluation of the single cervical skin test and interferon gamma responses to detect $\mathrm{Myco}$ bacterium bovis infected cattle in a herd co-infected with Mycobacterium avium subsp. paratuberculosis. Vet. Microbiol. 2014; 171(1-2):139-46. Disponible en: https://doi.org/10.1016/j.vetmic.2014.03.035.

[56] Estrada CC, Díaz OF, Arriaga DC, Villegas SN, Pérez GR, González SD. Concordancia de la PCR y métodos rutinarios para el diagnóstico de tuberculosis bovina. Vet. Méx. 2004; 35(3): 225-236.

[57] Retamal P, Abalos P. Comparación del ensayo de interferón gamma bovino con técnicas tradicionales para el diagnóstico de infección con Mycobacterium bovis en la Región Metropolitana de Chile. Rev. Colomb. Cienc. Pec. 2004; 17(3):223-230.

[58] Casal C, Díez GA, Álvarez J, Rodríguez CS, Mateos A, Linscott R, Martel E, et al. Strategic use of serology for the diagnosis of bovine tuberculosis after intradermal skin testing. Vet. Microbiol.2014; 170 (3-4):342-51. Disponible en: https://doi.org/ 10.1016/j.vetmic.2014.02.036.

[59] Organización Mundial de Sanidad Animal [Internet]. Paris: OIE; 2016. Manual de animales terrestres. Tuberculosis Bovina.; [citado 15 agost 2017]. Disponible en: http://www.oie.int/fileadmin/Home/ esp/Health_standards/tahm/2.04.06_BOVINE_ TB.pdf

[60] Sheridan MP, Browne JA, Doyle MB, Fitzsimons T, McGill K, Gormley E. IL-10 suppression of IFN- $\gamma$ responses in tuberculin-stimulated whole blood from Mycobacterium bovis infected cattle. Vet. Immunol. Immunopathol. 2017; 189:36-42. Disponible en: https://doi.org/10.1016/j.vetimm.2017.06.003.

[61] Vordermeier M, Gordon V, Hewinson G. Mycobacterium bovis antigens for the differential diagnosis of vaccinated and infected cattle. Vet. Microb. 2011; 151 (1-2): 8-13. Disponible en: https://doi. org/10.1016/j.vetmic.2011.02.020. 
[62] CasalC, Bezos J,Díez-Guerrier A, ÁlvarezJ, Romero B, de Juan L, Rodríguez-Campos S, et al. Evaluation of two cocktails containing ESAT-6, CFP-10 and Rv$3615 \mathrm{c}$ in the intradermal test and the interferon- $\gamma$ assay for diagnosis of bovine tuberculosis. Prev. Vet. Med. 2012; 105(1-2):149-54. Disponible en: https:// doi.org/ 10.1016/j.prevetmed.2012.02.007.

[63] Meloa ES, Souzaa II, Ramosb CA, Osóriob AL, Verbisckc NV, Araújoc FR. Evaluation of the use of recombinant proteins of Mycobacterium bovis as antigens in intradermal tests for diagnosis of bovine tuberculosis. Arch. Med. Vet. 2015. 47(3): 56-62. Disponibleen:http://dx.doi.org/10.4067/S0301-732 X2015000300003

[64] Lamont EA, Janagama HK, Ribeiro LJ, Vulchanova L, Seth M, Yang M, et al. Circulating Mycobacterium bovis peptides and host response proteins as biomarkers for unambiguous detection of subclinical infection. J. Clin. Microbiol. 2014; 52(2):536-43. Disponible en: http://dx.doi.org/10.1128/JCM.024 33-13

[65] Marassi CD, Medeiros L, McNair J, Lilenbaum W. Use of recombinant proteins MPB70 or MPB83 as capture antigens in ELISAs to confirm bovine tuberculosis infections in Brazil. Acta Tropical. 2011; 118(2): 101-104. Disponible en: https://doi. org/10.1016/j.actatropica.2011.02.015.

[66] Brett T, Tod S, Om S, Nelson J, Suelee R, Smith N, Desautels L, et al. Investigation of the cause of geographic disparities in IDEXX ELISA sensitivity in serum samples from Mycobacterium bovis-infected cattle. Sci Rep. 2016; 6: 22763. Disponible en: https://doi.org/ 10.1038/srep22763

[67] Waters WR, Buddle BM, Vordemeier HM, Gormley E, Palmer MV, Thacker JP. Development and evaluation of an enzyme-linked immunosorbent assay for use in the detection of bovine tuberculosis in cattle. Clin Vaccine Immunol. 2011; 18(11):1882-1888. Disponible en:https://doi.org/10.1128/CVI.05343-11.

[68] Rhodes G, McKinna C, Steinbach S, Bernardo D, Whelan C, Pirson G, Derek C. et al. Use of antigen-specific interleukin-2 to differentiate between cattle vaccinated with Mycobacterium bovis BCG and cattle infected with M. bovis. Clin. Vaccine Immunol. 2014; 21(1): 39-45. Disponible en: https:// doi.org/10.1128/CVI.00522-13

[69] Waters WR, Maggioli MF, Palmer MV, Thacker TC, McGill JL, Vordermeier HM, Berney-Meyer L, Jacobs WR, Jr, Larsen MH. Interleukin-17A as a biomarker for bovine tuberculosis. Clin. Vaccine Immunol. 2016. 23: 168-180. Disponible en: https:// doi.org/10.1128/CVI.00637-15

[70] Jeon HS, Shin AR, Son YJ, Kim JM, Jang Y. An evaluation of the use of immunoglobulin A antibody response against mycobacterial antigens for the diagnosis of Mycobacterium bovis infection in cattle. J. Vet. Diagn. Invest. 2015; 27(3):344-51. Disponible en: https://doi.org/10.1177/1040638715578879

[71] Fernández RD, Tavares L, Almeida da Silva PE, Dellagostin OA. Molecular typing of Mycobacterium bovis isolates: A review. Braz. J. Microbiol. 2014; 45(2): 365-372. Disponible en: http://dx.doi. org/10.1590/S1517-83822014005000045

[72] Costa P, Amaro A, Ferreira AS, Machado D, Albuquerque T. Rapid identification of veterinary-relevant Mycobacterium tuberculosis complex species using 16S rDNA, IS6110 and regions of difference-targeted dual-labelled hydrolysis probes. J. Microbiol. Meth. 2014; 107:13-22. Disponible en: https://doi.org/10.1016/j.mimet.2014.08.017

[73] Monteserin J, Paul R, Lopez B, Cnockaert M, Tortoli E, Menéndez C, García MJ, et al. Combined approach to the identification of clinically infrequent non-tuberculous mycobacteria in Argentina. Int $\mathrm{J}$ Tuberc Lung Dis. 2016; 20(9):1257-62. Disponible en: https://doi.org/10.5588/ijtld.16.0122

[74] Shimizu E, Macías A, Paolicchi F,Magnano G, Zapata L, Fernández A, et al. Genotyping Mycobacterium bovis from cattle in the Central Pampas of Argentina: temporal and regional trends. Mem. Inst. Oswaldo Cruz. 2014; 109(2): 236-245. Disponible en: http://dx.doi.org/10.1590/0074-0276140292.

[75] Suazo F, Casanova L, Torres C, Alarcón GJ, Reyes $\mathrm{J}$, Sosa S, Pezzat M, et al. Diversidad genética y distribución regional de cepas de Mycobacterium bovis del ganado en México. Rev. Mex. Cien. Pec. 2012; 3(4):459-471.

[76] Alzamora FF, Sidra EG, Harrison MV, Gomes MP, Cavalcante PN, Suffys JN. Múltiplas estirpes de isolados de Mycobacterium bovis identificados por tipagem molecular em bovinos abatidos em matadouros-frigoríficos. Pesq. Vet. Bras. 2014; 34(2): 103-108. Disponible en: http://dx.doi.org/10.1590/ S0100-736X2014000200001

[77] Jojoa-Jojoa J, Wintaco M, Osorio R, Puerto C, Guerrero G. First approach to molecular epidemiology of bovine tuberculosis in Colombia. Rev. MVZ Córdova. 2016; 21(1): 5222-5236. Disponible en: http:// dx.doi.org/10.21897/rmvz.32

[78] Yang L, Wang C, Wang H, Meng Q, Wang Q. Evaluation of MIRU-VNTR for typing of Mycobacterium bovis isolated from Sika deer in Northeast China. BMC Vet. Res. 2015; 11: 93-97. Disponible en: https://doi.org/10.1186/s12917-015-0402-0

[79] Martínez-Vidal C, Hori-Oshima S, De la Mora-Valle A, Bermúdez-Hurtado RM, Rentería-EvangelistaT, López V, Galván-Larac, L, Medina-Basulto G. Genotipificación por VNTR de aislados de Mycobacteri- 
um bovis de ganado sacrificado en Baja California, México. Rev Mex Cienc Pecu. 2011; 2(4): 393-401.

[80] Tejeda R, Arriaga DC Gilberto, Guevara VJ, García SJ, Torres LR, Estrada CC. Confirmación de la excreción de Mycobacterium bovis en exudados nasales mediante PCR anidada en un hato lechero. Vet. Méx. 2006; 37 (1): 137-143.

[81] King HC, Murphy A, James P, Travis E, Porter D. Performance of a noninvasive test for detecting Mycobacterium bovis shedding in european badger (Meles meles) Populations. J. Clin. Microbiol. 2015; 53(7): 2316-2323. Disponible en: http://dx.doi.org/ 10.1128/JCM.00762-15.

[82] Mittal M, Chakravarti S, Sharma V, Sanjeeth BS, Churamani CP, Kanwar NS. Evidence of presence of Mycobacterium tuberculosis in bovine tissue samples by multiplex PCR: possible relevance to reverse zoonosis. Transbound. Emerg. Dis. 2014; 61(2): 97-104. Disponible en: http://dx.doi.org/10.1111/ tbed.12203

[83] Sales ML, Fonseca AA, Orzil L, Alencar AP, Hodon MA, Issa MA, Soares Filho PM, Silva MR, Lage AP, Heinemann MB. Validation of two real-time PCRs targeting the PE-PGRS 20 gene and the region of difference 4 for the characterization of Mycobacteriumbovisisolates. Genet. Mol.Res. 2014;13(2):460716. Disponible en: http://dx.doi.org/10.4238/2014. June. 18.3
[84] Ikuta C, Carvalho R, Oliveira S, Francisco S, Henrique $\mathrm{H}$, Bryan $\mathrm{H}$, y Soares F. Comparison of DNA extraction protocols to detect Mycobacterium bovis in bovine tissue by PCR. Sem Ciên Agrár. 2016; 37(5): 3709-3718. Disponible en: http://dx.doi. org/10.5433/1679-0359.2016v37n5Supl2p3709

[85] Peled N, Ionescu R, Nol P, Barash O, McCollum M, VerCauteren K, Koslow M, Stahl R, Rhyan J, Haickb H. Detection of volatile organic compounds in cattle naturally infected with Mycobacteriumbovis. Sens. Actuat. 2012; 171-172: 588-94. Disponible en: https://doi.org/10.1016/j.snb.2012.05.038

[86] Ellis CK, Rice S, Maurer D, Stahl R, Waters WR, Palmer MV, Nol P, Rhyan R, VerCauteren K, Koziel J. Use of fecal volatile organic compound analysis to discriminate between non vaccinated and BCG vaccinated cattle prior to and after Mycobacterium bovis challenge. PloS One. 12(7): e0179914. Disponible en: https://doi.org/10.1371/journal.pone.0179914 